\section{Botulinum toxin as an initial therapy for management of sixth nerve palsies caused by nasopharyngeal carcinomas}

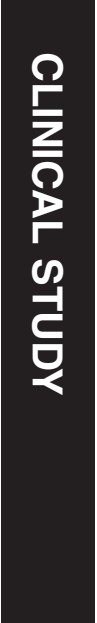

${ }^{1}$ Hong Kong Eye Hospital, Kowloon, Hong Kong

${ }^{2}$ Department of Ophthalmology and Visual Science, The Chinese University of Hong Kong, Shatin, Hong Kong

Correspondence: C Lam or J Yam, Hong Kong Eye Hospital, 147K, Argyle Street, Kowloon, Hong Kong

Tel: +852 2762 3000;

Fax: +852 27159490 .

E-mail: lamposang@gmail. com or yamcheuksing@ cuhk.edu.hk

Received: 3 April 2017 Accepted in revised form: 8 September 2017 Published online: 26 January 2018

\author{
Abstract \\ Purpose The purpose of this study is to \\ evaluate the efficacy and safety of botulinum \\ toxin injection as a primary treatment for \\ strabismus in a cohort of patients with \\ nasopharyngeal carcinoma (NPC)-related \\ chronic sixth nerve palsy. \\ Patients and methods We retrospectively \\ reviewed all cases of NPC-related sixth nerve \\ palsy receiving botulinum toxin injection in \\ the Hong Kong Eye Hospital between \\ January 2009 and January 2016. Only cases \\ with diplopia for at least 6 months; and failed \\ a trial of Fresnel prism therapy were \\ recruited. We excluded cases with prior \\ strabismus surgery and multiple cranial nerve \\ palsies. Patients were offered botulinum toxin \\ injection as primary treatment for their \\ strabismus and were given further injections \\ or offered surgery if diplopia persisted. \\ Success with botulinum toxin was defined as \\ a final distant orthophoria of $<15$ PD in \\ primary gaze, no diplopia in primary \\ position, and no head turn, as measured \\ 6 months after the last injection, without \\ requiring a second treatment. \\ Results A total of 25 patients were included \\ in the study. All patients received concurrent \\ chemo-radiotherapy for NPC. There was a \\ statistically significant reduction in the mean \\ deviation at distant after the last injection \\ compared to that at presentation $(P<0.001$, \\ Wilcoxin signed rank test). Overall, 7 patients \\ $(28 \%)$ achieved clinical success and 15 \\ patients $(64 \%)$ remained diplopia-free by \\ repeated botulinum toxin injections alone. \\ Nine patients went on to receive definitive \\ surgery and all achieved good ocular \\ alignment after surgery. Transient ptosis or \\ vertical deviation was seen in 7 patients, \\ which resolved within 3 months and no
}

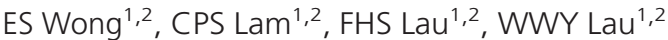
and JCS Yam ${ }^{1,2}$ serious complications arose from the treatment in our series.

Conclusions Botulinum toxin injection is a relatively less-invasive alternative to surgery that can be done under a topical anesthesia setting, which improves patient's quality of life via reduction in diplopia. It is a recommendable initial option in patients with chronic nerve palsies who may have higher risks associated with strabismus surgery. Eye (2018) 32, 768-774; doi:10.1038/eye.2017.276; published online 26 January 2018

\section{Introduction}

Nasopharyngeal carcinoma (NPC) is endemic to Southeast Asia. It is one of the top 10 most common cancers and is a major cause of cancer deaths in Hong Kong. ${ }^{1}$ The mainstay of treatment is radiotherapy or concurrent chemoradiotherapy (CCRT). ${ }^{2}$ Advancement in imaging and treatment modalities has resulted in a decline in the total mortality of the disease. ${ }^{3}$ However, diplopia caused by cranial nerves dysfunction, which occurs in about $1-5 \%$ of NPC patients, still remains as a major source of disturbance to quality of life. ${ }^{4,5}$ Cranial nerves impairment can be caused by local invasion of tumor to the skull base and cavernous sinus; or is related to post-radiotherapy complications. Among them, sixth nerve palsy is relatively common, because of its proximity to skull base and its small size. ${ }^{6}$

Despite shielding of the eyes during radiotherapy, post-irradiation ischemic changes can occur not only in the form of radiation optic neuropathy or retinopathy but even in a delayed manner as an increased risk of anterior segment ischemia with routine strabismus surgery. ${ }^{7}$ 
Cranial neuropathies after NPC treatment such as bulbar nerve palsy; ${ }^{8}$ and poor systemic comorbidity would also increase the risk of general anaesthesia in this group of patients. Very often, this group of patients would prefer conservative management whenever possible.

Botulinum toxin injection into the medial rectus muscle in sixth nerve palsy has been proposed as an effective alternative to surgery. It has been shown to bring temporary relief of symptoms or even an increase in recovery rate of patients with sixth nerve palsy from many causes. ${ }^{9}$ However, the efficacy of botulinum toxin injection for strabismus secondary to nerve palsy caused by tumors, in particular NPC, has not been reported extensively. We observed that botulinum toxin injection should be a good alternative to improve quality of life in NPC patients who are not fit or not willing for strabismus surgery. We therefore reported our experience with botulinum toxin injection as a primary initial treatment for chronic sixth nerve palsy secondary to NPC or its treatment-related complications.

\section{Materials and methods}

This is a retrospective chart review, observational case series. All consecutive patients receiving botulinum toxin injection in Hong Kong Eye Hospital were identified between January 2009 and January 2016. Inclusion criteria were as follows: (1) sixth nerve palsy resulted from NPC and its related treatment; (2) duration of symptoms for more than 6 months; (3) no prior history of strabismus; (4) diplopia at primary gaze at $6 \mathrm{~m}$; (5) failure of Fresnel prism therapy; and (6) in remission and not on active treatment for underlying NPC. Patients suffered from multiple cranial nerve palsies, or those with follow-up after botulinum toxin injection $<6$ months were excluded.

The following data were collected for analysis: date of birth; laterality; date of diagnosis for NPC; age at tumor diagnosis; date of surgery for tumor; type of treatment; date of onset of the sixth nerve palsy; date of the first eye examination; date of the last eye examination; angle of deviation at $6 \mathrm{~m}$ in the primary position was recorded in prism diopters both before intervention and at follow-up after each intervention. Monocular abduction deficit was recorded on a scale described by Scott and Kraft: ${ }^{10}$ 0 (normal); - 1 (to $75 \%$ full rotation); - 2 (to $50 \%$ full rotation); -3 (to $25 \%$ full rotation); -4 (to midline); and -5 (inability to abduct to the midline). The date of botulinum toxin treatment, number of botulinum toxin injections, and any strabismus surgeries received were all retrieved.

\section{Definition}

Treatment success for sixth nerve palsy was defined as an esophoria $<15$ PD in primary gaze, no diplopia in primary position at distant fixation, and no abnormal head posture at the end of follow-up period (at least 6 months after receiving the last injection). Treatment failure was defined as failure to achieve the above goals or the need of a second intervention or change in management to achieve the above goals.

\section{Surgical technique}

Botulinum toxin (Botox onabotulinumtoxinA, Allergan, Dublin, Republic of Ireland) injection was done under topical anesthesia. A unit of $100 \mathrm{U}$ botulinum toxin was reconstituted with $4 \mathrm{ml}$ saline to produce $2.5 \mathrm{U}$ botulinum toxin in $0.1 \mathrm{ml}$ for the preparation. Dosage of injection depends on the angle of deviation: (1) 1.25-2.5 U to one muscle if the deviation was $<20$ PD; and (2) 2.5-5.0 U to one or two muscles (depending on prism diopters and surgeon preference) if the deviation was ranging at 20-50 PD. Under electromyography guidance (Clavis, The Dantec Clavis device, distributed by Natus Medical Incorporated, Pleasanton, California, United States), botulinum toxin was injected with a 27-gauge, 1.5-inch needle at $\sim 1 \mathrm{~cm}$ posterior to the insertion of the medical rectus muscle on the globe. All patients received tobrex (tobramycin $3 \mathrm{mg} / \mathrm{ml}$, Alcon, Fort Worth, Texas, USA) eye drops one drop every $6 \mathrm{~h}$ for 1 week postoperatively. Patients were followed up at 1 week, 2 months, and 6 months after each injection.

\section{Statistical analysis}

Statistical analyses were performed using SPSS version 22.0 (SPSS, Inc., Chicago, IL, USA). Descriptive statistics of primary and secondary outcome measures in both groups were reported. Group median was compared with Wilcoxon rank sum test for independent data and Wilcoxon signed ranks test for paired data. Success rates were evaluated using Fisher's exact test. A $P$-value of $<0.05$ was considered statistically significant.

The study was approved by the local institutional research board and was conducted in accordance with the Declaration of Helsinki.

\section{Results}

A total of 25 patients were recruited during the study period. All patients had been treated for NPC with either radiotherapy or CCRT. There was a male predominance (male : female $=22: 3$ ) consistent with data from the HK Cancer Registry. ${ }^{1}$ Mean age at presentation was 
$53.3 \pm 8.84$ years (range $35-69$ years). The mean followup duration from presentation to last follow-up was 39 months (range 8 months -7 years). By the end of the follow-up period 11 patients had passed away due to coexisting systemic disease.

All patients had diplopia. The sixth nerve palsies were bilateral in 11 patients and unilateral in 14. The mean of primary deviation at distance was $39.3 \pm 16.4 \mathrm{PD}$ (range 10-80 PD) at presentation. All patients failed a previous trial of Fresnel prism therapy. Among all patients, 8 of them received botulinum toxin injection as initial management because they were not fit for strabismus surgery due to poor comorbid condition. The remaining 17 patients were physically fit for surgery, but they all preferred botulinum toxin therapy as initial treatment. Patient demographics and treatment details are listed in Table 1.

\section{Overall effect of botulinum toxin}

Patients received a mean of $3 \pm 3.0$ injections (range $1-12$ ). The mean of primary deviation was $33.3 \pm 20.8$, $31.3 \pm 14.7$, and $28.08 \pm 19.34$ PD at 6 months after the first injection, 6 months after second injection, and 6 months after third injection, respectively. There was a statistically significant reduction in the mean deviation at distant after the last injection compared to presentation $(P<0.001$, Wilcoxin signed rank test).

Reduction in angle of deviation after each injection After the first injection, an immediate improvement was seen with the mean reduction in angle of deviation of 22.6 \pm 12.9 PD at 1 week postoperatively. In all, 19 out of 25 patients reported free of diplopia within 2 months after the first injection. However, the results worn off by 6 months postoperatively with a mean reduction in angle of deviation dropping to $5.87 \pm 6.47$. A total of 12 patients went on to receive the second botulinum toxin injection with reduction in the angle of deviation being $24.8 \pm 11.4$ PD at 2 months post injection. By 6 months post injection the results weaned off with a mean reduction in angle of deviation of $6 \pm 5.9$ PD. Similar figures were seen after the third injection where the mean reduction in angle of deviation was $27.2 \pm 14.0$ and $6.2 \pm 6.49 \mathrm{PD}$ at 1 week and 6 months after operation, respectively.

Treatment success Out of the 25 patients, 16 cases (64\%) remained diplopia-free by repeated botulinum toxin injections alone. Among them, 7 cases (28\%) fulfilled the definition of clinical success. The remaining 9 cases eventually required strabismus surgery after failure of botulinum toxin therapy. Among these 9 patients, 7 had angles of deviation $>30$ PD on presentation, and had poor response to botulinum toxin injection. They all received muscle transposition surgeries as the definite treatment. The remaining 2 patients had moderate deviation of 15-30 PD on presentation and had partial response to botulinum toxin. They received MR recession surgery alone. All 9 cases achieved good ocular alignment after surgery. On the other hand, the remaining 9 patients who did not fulfill the criteria for treatment success declined strabismus surgery and opted for repeated botulinum toxin injections to maintain diplopia-free status.

Complications Transient ptosis (resolved within 3 months) was seen in four patients after the first injection, but none in subsequent injections despite botulinum toxin therapy at same dosage. Transient vertical deviation $<6$ PD (resolved within 3 months) was seen in another three patients after the first injection. No serious complications were reported in our series.

\section{Preoperative and intraoperative factors for treatment} success We performed univariate analysis to compare the differences in characteristics between the success group with botulinum toxin therapy alone and nonsuccess group (Table 2). Preoperative deviation was significantly lower in the success group compared with non-success group $(26.00 \pm 11.0$ PD vs $44.4 \pm 15.4, P=0.01$, Wilcoxon rank sum test). The corresponding dosage of botulinum toxin therapy was also lower in the success group compared with non-success group, which approached statistical significance $(3.29 \pm 1.19$ vs $4.58 \pm 0.96, P=0.05$, Wilcoxon rank sum test). There was no significant difference between groups in terms of age of presentation, duration of palsy, EOM function, or number of injections received.

\section{Discussion}

We reported the efficacy of botulinum toxin injection as initial therapy in a cohort of chronic sixth nerve palsy secondary to NPC. Notably, there was persistent reduction in angle of deviation after repeated botulinum toxin injections. About $28 \%$ and $64 \%$ of patients can achieve clinical success and diplopia-free by repeated botulinum toxin injections, respectively. Patient with a smaller preoperative angle of deviation has a higher chance of treatment success. NPC is endemic in our locality, and that this group of patient has an increased risk of strabismus surgery because of poor systemic comorbidity. Therefore, sparing these patients from surgical therapy is important and highly relevant.

Botulinum toxin was first used for the treatment of strabismus in the early 1980s. Over the past 20 years, its use in strabismus has rapidly expanded to include the treatment of various nerve palsies, dysthyroid strabismus, and augmentation of strabismus surgery, etc. ${ }^{11}$ The effect of botulinum toxin on muscle paralysis is believed to be 


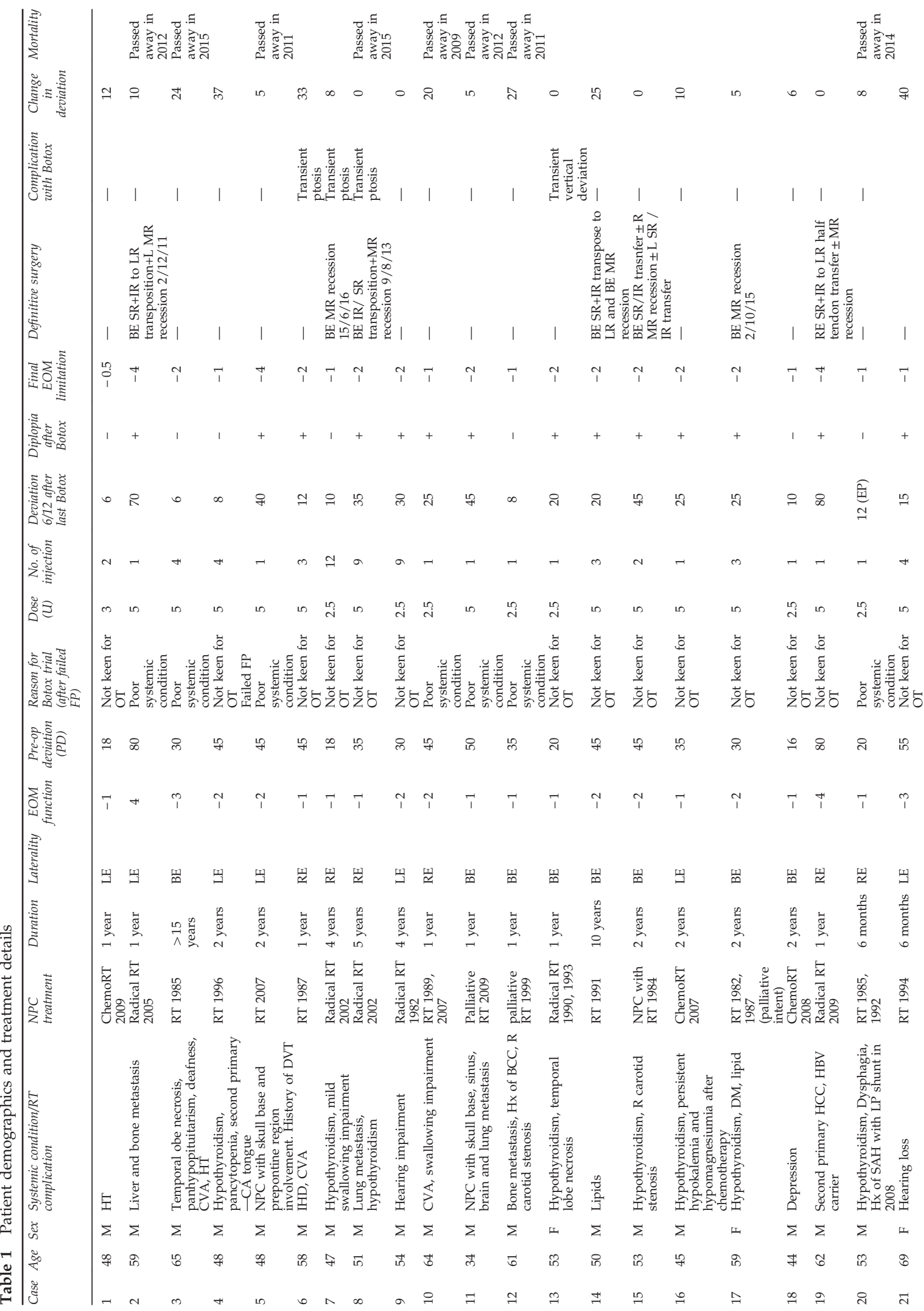




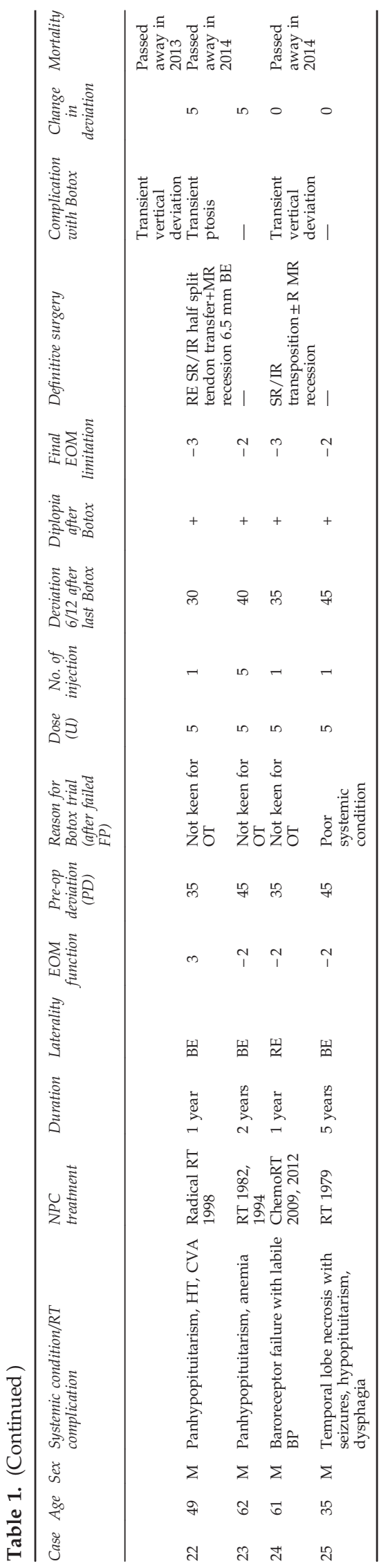

Table 2 Univariate analysis to compare the differences in characteristics between the success group with botulinum toxin therapy alone and non-success group

\begin{tabular}{lccc}
\hline & No success (18) & Success (7) & P-value $^{\mathrm{a}}$ \\
\hline Age (year) & $53.67 \pm 9.38$ & $52.29 \pm 7.87$ & 0.46 \\
$\begin{array}{l}\text { Duration of palsy } \\
\text { (year) }\end{array}$ & $2.36 \pm 2.35$ & $3.64 \pm 5.137$ & 0.88 \\
Pre-op deviation & $44.4 \pm 15.42$ & $26.00 \pm 10.97$ & 0.01 \\
EOM function & $2.11 \pm 0.90$ & $1.43 \pm 0.79$ & 0.09 \\
Number of injection & $2.67 \pm 2.61$ & $3.57 \pm 3.95$ & 0.57 \\
Dosage & $4.58 \pm 0.96$ & $3.29 \pm 1.19$ & 0.05 \\
\hline
\end{tabular}

${ }^{a}$ Mann-Whitney U-test.

transient, which is typically seen within 2-4 days and is expected to wean off 6-12 weeks after injection. However, some possible long-term effects of botulinum toxin have been reported, leading to its use as a sole treatment for strabismus by others. ${ }^{12}$

The proposed mechanism of botulinum toxin in chronic nerve palsy, as compared to prevention of muscle contracture in acute nerve palsies, has been postulated to be related to a structural alteration in the rectus muscle produced by chemodenervation. The animal model by Spencer and McNeer ${ }^{13}$ revealed a permanent reduction of muscle fiber cross-sectional area in injected muscles compared to non-injected ones. This would lead to a permanent change in the muscular length tension curves and fatigue characteristics, resulting in change in ocular alignment, even after the wean off of botulinum toxin effect. This after-effect is believed to be augmented by the presence of motor fusion. ${ }^{11}$ Our study concurred with previous reports on the after-effect of botulinum toxin in cases of chronic nerve palsy. We demonstrated a significant smaller angle of deviation at least 3 months after the last injection of botulinum toxin when compared with that at presentation. Although this effect alone could not lead to clinical success in majority of cases, our reported of $28 \%$ success should also serve as an important reference to eligible patients. Similarly, Repka et al ${ }^{14}$ and Biglan et al ${ }^{15}$ conducted studies on the use of botulinum toxin in the initial management of chronic sixth nerve palsies. At 6 months of follow-up after last injection, $41 \%$ and $64 \%$ of patients in their studies showed at least $50 \%$ reduction in angle of deviation, respectively. In both studies, botulinum toxin therapy was not as effective as surgery, but was still encouraging. On the other hand, another prospective study by Holmes et al ${ }^{16}$ reported that only 1 out of 10 patients with chronic sixth nerve palsy was successfully managed with botulinum toxin alone. However, it is difficult to directly compare the success rates from various studies due to different methodology and definitions of success. The relatively low success from the study by Holmes et al ${ }^{16}$ concluded that botulinum 
toxin alone is less likely to produce promising results in chronic sixth nerve palsy and that at least two procedures are necessary to regain single binocular vision in this group. In our series, nine cases eventually required strabismus surgery after poor response to botulinum toxin therapy. Among them, seven cases with > 30 PD presenting deviation with poor response to botulinum toxin treatment received transposition surgery. However, in two cases with partial response to botulinum toxin but whom still complained of diplopia, we were able to achieve good clinical outcome with a simpler single muscle MR recession procedure. This highlights another benefit of an initial botulinum toxin trial-even if monotherapy with botulinum toxin could not bring about treatment success, presence of partial response, or possible resultant reduction in overall deviation after the therapy may prompt the choice of a simpler recession procedure over a more complicated transposition surgery, which would involve operation on more muscles and consequently incur higher risk.

A previous study of 95 patients undergoing at least eight botulinum toxin injections for strabismus demonstrated an overall trend of fewer injections required with time. ${ }^{17}$ However, our study did not demonstrate an association between the number of botulinum toxin injection and treatment success. In addition, increase in dosage of botulinum toxin per injection also did not lead to increase in success. In contrary, we observed that the dosage of botulinum toxin was lower in the success group comparing to the nonsuccess group (3.29 vs $4.58 \mathrm{U}$ ). This could be explained by the fact that lower dosage of botulinum toxin is required for smaller preoperative angles of deviation, which is an important factor for treatment success. Horgan et al ${ }^{17}$ reported no adverse outcomes from their long-term botulinum toxin therapy, concluding that botulinum toxin therapy is an appropriate and safe option for strabismus patients who do not want to undergo further surgery. Transient ptosis or vertical deviation was observed in seven cases with the first injection, the occurrence was consistent with literature. ${ }^{18}$ No serious ocular or systemic complications from the botulinum toxin intervention was reported in our series.

Importantly, we have demonstrated that a smaller preoperative deviation may lead to higher treatment success with botulinum toxin alone. The 'after-effect' of botulinum toxin on medial rectus was postulated to be dependent on the opposing force from the lateral rectus, which would be absent in complete sixth nerve palsy. ${ }^{11}$ Hence, we believe that the best outcomes for botulinum toxin injection in chronic sixth nerve palsy are those with partially recovered or fully recovered lateral rectus function, with mild contracture of the medial rectus, and mild to moderate deviation (15 - 30 PD) at primary. In our series, other factors including age of presentation and duration of the chronic nerve palsy did not influence the success.

Despite the improving life expectancy, NPC survivors continue to suffer from long-term complications or side effects of radiotherapy or CCRT. ${ }^{19}$ In our series, 8 patients were deemed systemically unfit to tolerate a major surgery, and by the time of conclusion of this study, 11 patients passed away due to systemic diseases. Botulinum toxin, being a relatively simple and less-invasive procedure with a low complication rate, is a good initial therapy in the management of diplopia and esodeviation in patients with debilitating systemic conditions or those at a higher risk of squint surgery.

Our study is limited by its retrospective nature. In addition, our sample size is relatively small, which might affect the power of our statistical analysis. Nevertheless, our cohort is the largest report of its kind in the literature.

\section{Conclusion}

Botulinum toxin is a recommendable initial option in patients with chronic sixth nerve palsy secondary to NPC, who are at a higher risk of anterior segment ischemia with strabismus surgery ${ }^{7}$ and whose nerve palsy has a lower chance of spontaneous recovery. It is a relatively lessinvasive alternative to surgery and facilitates earlier regaining of binocularity and reduction of diplopia, which may improve patient's quality of life.

\section{Summary}

What was known before

- Botulinum toxin injection into the medial rectus muscle in sixth nerve palsy has been proposed as an effective alternative to surgery.

- Temporary relief of symptoms, increase in recovery rate, and even a longer-lasting 'after-effect' have been reported in its use in strabismus from many causes.

- However, the efficacy of botulinum toxin injection for strabismus secondary to nerve palsy caused by tumors, in particular nasopharyngeal carcinoma, has not been reported extensively.

What this study adds

- This is the largest cohort of nasopharyngeal carcinomarelated chronic sixth nerve palsy cases in literature.

- Botulinum toxin is a recommendable initial option in this group of patients whose nerve palsy has a lower chance of spontaneous recovery. They often have poor general condition and are at a higher risk of anterior segment ischemia with strabismus surgery.

- Botulinum toxin injection is a relatively less-invasive alternative to surgery that facilitates earlier regaining of binocularity and reduction of diplopia, which may improve patient's quality of life. 


\section{Conflict of interest}

The authors declare no conflict of interest.

\section{References}

1 Hong Kong Cancer Registry, Hospital Authority. Available at http://www.chp.gov.hk/en/content/9/25/54.html (accessed on 30 June 2017).

2 Chua ML, Wee JT, Hui EP, Chan AT. Nasopharyngeal carcinoma. Lancet 2016; 387: 1012-1024.

3 Lee AW, Foo W, Mang O, Sze WM, Chappell R, Lau WH et al. Changing epidemiology of nasopharyngeal carcinoma in Hong Kong over a 20-year period (1980-99): an encouraging reduction in both incidence and mortality. Int J Cancer 2003; 103: 680-685.

4 Huang S, Chu G. Nasopharyngeal cancer: study II. Int J Radiat Oncol 1981; 7: 713-716.

5 Lee AW, Law SC, Ng SH, Chan DK, Poon YF, Foo W et al. Retrospective analysis of nasopharyngeal carcinoma treated during 1976-1985: late complications following megavoltage irradiation. Br J Radiol 1992; 65: 918-928.

6 Chong VF, Khoo JB, Chan LL, Rumpel H. Neurological changes following radiation therapy for head and neck tumours. Eur J Radiol 2002; 44: 120-129.

7 Yip WW, Yu CB, Fan DS, Yick DW, Rao SK, Lam DS. Anterior segment ischemia after two-muscle surgery in a patient with radiation-treated nasopharyngeal carcinoma. J Pediatr Ophthalmol Strabismus 2008; 45: 40-42.

8 Lin YS, Jen YM, Lin JC. Radiation-related cranial nerve palsy in patients with nasopharyngeal carcinoma. Cancer 2002; 95: 404-409.

9 Elston JS, Lee JP. Paralytic strabismus: the role of botulinum toxin. Br J Ophthalmol 1985; 69: 891-896.
10 Scott AB, Kraft SP. Botulinum toxin injection in the management of lateral rectus paresis. Ophthalmology 1985; 92: 676-683.

11 Kowal L, Wong E, Yahalom C. Botulinum toxin in the treatment of strabismus. A review of its use and effects. Disabil Rehabil 2007; 29: 1823-1831.

12 Scott AB. Botulinum toxin injection into extraocular muscles as an alternative to strabismus surgery. Ophthalmology 1980; 87: 1044-1049.

13 Spencer RF, McNeer KW. Botulinum toxin paralysis of adult monkey extraocular muscle. Structural alterations in orbital, singly innervated muscle fibers. Arch Ophthalmol 1987; 105: 1703-1711.

14 Repka MX, Lam GC, Morrison NA. The efficacy of botulinum neurotoxin A for the treatment of complete and partially recovered chronic sixth nerve palsy. J Pediatr Ophthalmol Strabismus 1994; 31: 79-83.

15 Biglan AW, Repka MX, GC Lam, Morrison NA. The efficacy of botulinum neurotoxin A for the treatment of complete and partially recovered chronic sixth nerve palsy. J Pediatr Ophthalmol Strabismus 1994; 31: 84.

16 Holmes JM, Leske DA, Christiansen SP. Initial treatment outcomes in chronic sixth nerve palsy. J AAPOS 2001; 5: 370-376.

17 Horgan SE, Lee JP, Bunce C. The long-term use of botulinum toxin for adult strabismus. J Pediatr Ophthalmol Strabismus 1998; 35(1): 9-16 quiz 44-15.

18 Committee on ophthalmic procedures assessment of the American Academy of Ophthalmology. Botulinum toxin therapy of eye muscle disorders. Safety and effectiveness. American Academy of Ophthalmology. Ophthalmology 1989; Suppl: $37-41$.

19 Lee CC, Ho CY. Post-treatment late complications of nasopharyngeal carcinoma. Eur Arch Otorhinolaryngol 2012; 269: 2401-2409. 